\title{
The Design of All Fiber Laser Heterodyne Detection System
}

\author{
Cao Miao ${ }^{1}$, Cui Wenjie ${ }^{* 1}$ andYao Qinghua ${ }^{2}$ \\ 1.Changchun University of Science and Technology, Jilin Changchun, 130022, \\ China, 2.Changchun University of Technology, Jilin Changchun, 130012, China \\ E-mail:79816540@qq.com
}

\begin{abstract}
The traditional method of heterodyne detection is improved in this paper based on the principle of laser heterodyne detection. The laser heterodyne detection system is designed with all-fiber laser radar in all fiber system, and the laser, the optical system, the receiving device and so on are connected via the passive component. The system can mix the local oscillation light with signal light of measured object information in the photodetector surface, and the difference frequency signal is obtained. Then the signal is filtered and amplified by the signal processing technique to eliminate the DC component, and the desired difference frequency signal is obtained. Through the analysis of the performance of all fiber laser heterodyne detection system, the system has a strong ability to detect weak signal, high conversion gain and signal to noise ratio, and has a good filtering effect. The system is very suitable for the application of laser ranging and so on.
\end{abstract}

Keywords: all fiber, laser, heterodyne, detection

\section{Introduction}

In the photoelectric detection system, the signal is usually required to be transformed. For example, the conversion between one light quantity and another light quantity; the conversion between non-light quantity and light quantity; the conversion between continuous light quantity and pulse light quantity. There are many kinds of classifications for the way of testing. According to the source of information, detection can be divided into active detection system and passive detection system. According to the relative area of the target, it can be divided into point detection system and surface detection system. According to the signal modulation method and signal processing method, it can be divided into analog detection system and coherent detection system. According to the way of light wave to carry the information signal, it can be divided into direct detection system and heterodyne detection system. In this paper, the optical signal of the target is converted into electrical signal, so heterodyne detection is used.

Based on the principle of differential frequency, optical heterodyne detection can mix the local oscillation light with signal light of measured object information in the photodetector surface, and the difference frequency signal is obtained. Then the signal is filtered and amplified to eliminate the DC component by the signal processing technique, and the desired difference frequency signal is obtained. The sensitivity of heterodyne detection is higher than that of direct detection, so it can greatly improve the sensitivity of the detection signal. At the same time, this method can enhance the interference ability of signal transmission, and improve the frequency selectivity of signal processing, which has a very strong advantage in terms of weak signal detection. 


\section{The Principle of Laser Heterodyne Detection}

The principle of the laser heterodyne detection technology is similar to the electronic heterodyne detection technique. The difference lies in the different carriers only. At present, laser heterodyne detection is mainly used in target detection of radar system, optical ranging, atmospheric remote sensing, optical tracking and other fields. The vibration light and the signal light are generated coherent in the photoelectric detector, light is used as the carrier. The principle of optical heterodyne detection is shown in Figure 1.

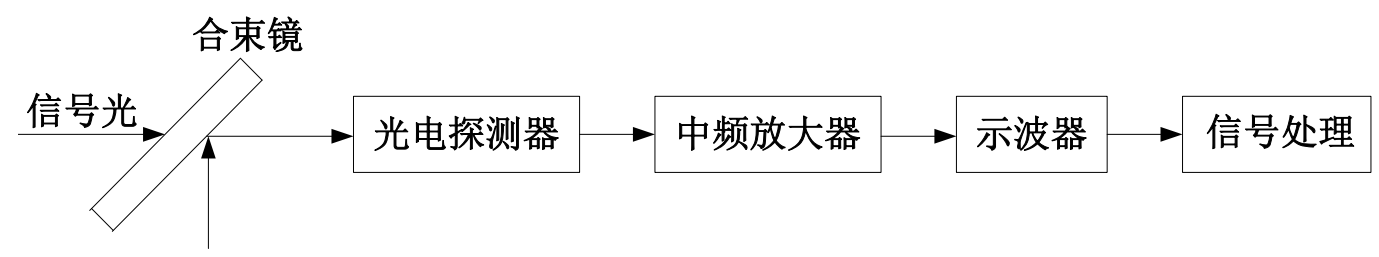

本振光

Figure 1. Block Diagram of Optical Heterodyne Detection

The basic principle of optical heterodyne detection is to extract difference frequency signal of two beams of light. The two beams of light are used as local oscillator as and signal light with the measured information. The ultimate goal is to measure the frequency difference of the two beams of light. The frequency difference is achieved by photomixing, so it is also called the intermediate frequency signal. The optical information contained in this intermediate frequency signal includes: frequency, amplitude, phase, etc And then through the electrical signal processing, you can get the relevant information of the target. At a certain time $t$, and at a point $\mathrm{R}$ on the inner surface of sensitive detector, the electromagnetic field of two beams of coherent light waves can be expressed by the complex exponential function as follows:

$$
\begin{aligned}
& E_{1}(r, t)=e_{1} A_{1}(r) \exp \left(i \omega_{1} t\right) \\
& E_{2}(r, t)=e_{2} A_{2}(r) \exp \left(i \omega_{2} t\right)
\end{aligned}
$$

Wherein, $e_{1}, e_{2}$ is a unit vector in the direction of polarization; $A_{1}(r), A_{2}(r)$ is the complex amplitude of the two beams of light; $\omega_{1}, \omega_{2}$ is the angular frequency of the light field. Wherein, the the value of complex amplitude determines the light intensity of the two beams, and the phase distribution of the space.

When the two beams of light react with each other, the light field is:

$$
E(r, t)=E_{1}(r, t)+E_{2}(r, t)
$$

Within the response area of photoelectric detector, if the synthesized light field abovementioned illuminates on the photosensitive surface $\sigma$ of the photodetector, the response photocurrent $\mathrm{i}(\mathrm{t})$ will be generated in a small facet element $\delta \sigma$ of this region. The facet element is located at a certain point $r$ on the inner surface of the detector. The pulse amplitude of the photoelectron current formed in the external circuit is:

$$
\delta i(r, t)=\frac{q}{h v} \eta(r) I(r, t) \delta \sigma
$$


Wherein, $\mathrm{q}$ is the amount of charge, $h v$ is the average photon energy of the two beams of interference light, $\eta(\mathrm{r})$ is the quantum efficiency of the photosensitive surface of the photodetector at the point $\mathrm{r}, I(r, t)=|E(r, t)|^{2}$ is the strength of interference field. Thus, $\delta i(r, t)$ can be integrated on the entire surface of the sensitive detector, and you can get the total output current of the photodetector:

$$
i(t)=\frac{q}{h v} \int_{\sigma} \eta(r) I(r, t) d^{2} r
$$

The interference of two light waves in the surface of the detector is shown in Figure 2.

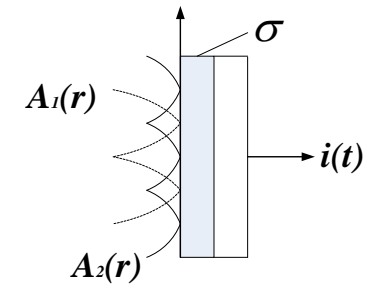

Figure 2. The Interference of Two Light Waves in the Surface of the Detector

Bringing the formula (1) and (2) into the formula (5):

$$
\begin{aligned}
& i_{1}(t)+i_{2}(t)+\frac{q}{h v} e_{1} \cdot e_{2} \exp \left[i\left(\omega_{1}-\omega_{2}\right) t\right] \int \eta(r) A_{1}(r) A_{2}^{*}(r) d^{2} r \\
& +\frac{q}{h v} e_{1} \cdot e_{2} \exp \left[-i\left(\omega_{1}-\omega_{2}\right) t\right] \int \eta(r) A_{1}^{*}(r) A_{2}(r) d^{2} r
\end{aligned}
$$

The first two items $i_{1}(t)$ and $i_{2}(t)$ in the form (6) are produced by $E_{1}(r, t)$ and $E_{2}(r, t)$ respectively, and they are constant photocurrent items which are timeindependent; * denotes a complex conjugate. Among them,

$i_{1}(t)=\frac{q}{h v} \int_{\sigma} \eta(r)\left|A_{1}(r)\right|^{2} d^{2} r$

$i_{2}(t)=\frac{q}{h v} \int_{\sigma} \eta(r)\left|A_{2}(r)\right|^{2} d^{2} r$

The third and fourth are to determine the resonant variation of the photocurrent with angular frequency $\left|\omega_{1}-\omega_{2}\right|$, and they is the interference portion of the two optical field.

In the following, a hypothesis in the simplest case is made. Suppose $\eta, A_{1}, A_{2}$ in the formula (7) and (8) are not related to the spatial coordinates $r$, the third and fourth of the formula (6) can be written as follows

$$
i_{I F}(t)=2 \frac{\eta q}{h v} \sigma e_{1} \cdot e_{2}\left|A_{1}\right|\left|A_{2}\right| \cos \left[\left(\omega_{1}-\omega_{2}\right) t+\Delta \varphi\right]
$$


The intermediate frequency signal is also known as the intermediate frequency signal, because it contains both the amplitude information of the interference field and the phase information, so it is the main research object of the laser heterodyne detection.

In the formula, $\sigma$ is the light-sensitive surface area of the photodetector, $\Delta \varphi$ is the phase difference of the complex amplitude $A_{1}$ and $A_{2} \cdot i_{I F}(t)$ is also known as the intermediate frequency signal. Since it not only includes the amplitude information of the interference field, but also contains the phase information, it has become the main subject of laser heterodyne detection.

Compared with the direct detection, the optical heterodyne detection technology can effectively enlarge the received optical signal. So the technology has a high sensitivity, which is also the most basic advantage. If now there is a target, the light field is $E_{1}$, the amplitude of the signal is $A_{1}$, but $A_{1}$ is very small. As can be seen in the formula (9), the current signal of intermediate frequency $i_{I F}(t)$ is determined by the product of the amplitude $\left|A_{1}\right|\left|A_{2}\right|$, and not just depend on $A_{1}$, in which $A_{2}$ is the vibration amplitude. Therefore, in theory, when $A_{1}$ is very small, as long as the amplitude of the vibration light $A_{2}$ is increased to be large enough, the output of the intermediate frequency signal can be increased to the level that matches the photoelectric detector.

\section{Laser Heterodyne detection System Design}

Figure 3 shows the specific design scheme of all-fiber laser lidar heterodyne detection system. All connecting lines in the figure are both polarization maintaining fibers, and each piece of optical fiber is connected through the polarization maintaining fiber adapter.

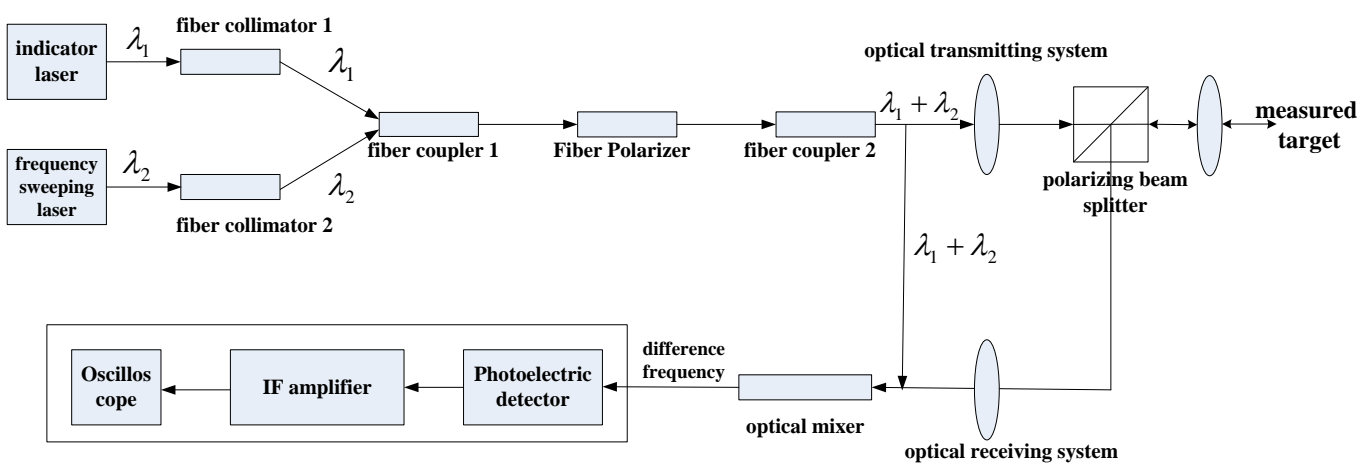

Figure 3. Laser Heterodyne Detection System

The red laser beam emitted from the indicator laser, whose wavelength $\lambda_{1}=730 \mathrm{~nm}$, is used as the indicator light. It turns the inside optical fiber transmission light into collimated light through the fiber collimator 1 . At the same time, the frequency sweeping laser exits the near infrared laser beam, whose wavelength $\lambda_{2}=1630 \mathrm{~nm}$, as the measuring light. It turns the inside optical fiber transmission light into collimated light through the fiber collimator 2 . The output light $\lambda_{1}$ and $\lambda_{2}$ of two wavelengths come into the fiber coupler 1 for transmission. The two laser beams of different wavelengths, which exit from the fiber coupler 1, get through the fiber polarizer. The polarized light is obtained in the fiber output end. Then through the fiber coupler $2, \lambda_{1}$ and $\lambda_{2}$ will be beam-splitted respectively. Because the measurement signal of the target is relatively weak, the coupling ratio of the coupler 2 should be 1/11, which can make the light energy 
ratio of the vibration light and measuring light become 1:9, in order to enhance the signal to noise ratio and improve the detection capability. The light emitted from the coupler 2, is partly used as the measuring light incident to the emitting optical system, and enters the polarizing beam splitter. The other part is used as the vibration light, and enters he optical mixer directly. The measuring light into a polarizing beam splitter can be split into two beams. One beam gets through the optical system incident to the measured surface of the target. The laser beam reflected from the surface of the target comes into the receiving optical system through polarization beam splitter, and it eventually enters the optical mixer. It mixes with the vibration light in the mixer, and the difference frequency signal of the vibration and measuring light are get, which is still output according to the original polarization direction.

\section{The Performance Analysis of Laser Heterodyne Detection System}

1) In favor of weak signal detection

The optical heterodyne detection system has a high sensitivity, which is is extremely beneficial for weak signal detection. As is shown in the formula (9), the product of the optical amplitude of the signal $A_{1}$ and the amplitude of the vibration light $A_{2}$ is $\left|A_{1} \cdot A_{2}\right|$, and the amplitude of the intermediate frequency signal $i_{I F}(t)$ is proportional to it. By the formula (9) shows that, while the amplitude is proportional to its IF photocurrent. Thus, when the light signal is weak, as long as the vibration light is strong enough, that is, the product of the two is large enough, you can achieve high detection requirements.

2) High conversion gain

The optical heterodyne detection system is to detect the frequency difference between the vibration light and the signal light, whose result is a medium frequency signal. That is, the optical heterodyne detection is to convert the optical frequency signal to an intermediate frequency current signal. This conversion process causes a kind of conversion gain of the optical heterodyne detection, and the essence is the role of the vibration light.

The specific value of the conversion gain is discussed. Based on the direct detection of the target, the conversion gain $\mathrm{G}$ is defined as :

$G=\frac{P_{I F}}{P_{S}}$

$P_{S}$ is the output optical power of the signal light, $P_{I F}$ is the output medium frequency power of the optical heterodyne detection.

$P_{I F}=2 \alpha^{2} P_{S} P_{L} R_{L}$

$\alpha$ is the scale factor, $P_{L}$ is the output optical power of the vibration light, $R_{L}$ is the Resistance of photoelectric detector.

$P_{L}=I_{S}^{2} R_{L}=\alpha^{2} P_{S}^{2} R_{L}$

Now unite the formula (10), (11) and (12):

$G=\frac{2 P_{L}}{P_{S}}$ 
As can be seen from the formula (13), the size of the conversion gain is related to the ratio of $P_{L}$ and $P_{S}$, and $P_{L}>>P_{S}$. The conversion gain calculated by the formula (13) is relatively high.

\section{3) Having a good filtering ability}

In the optical detection field, the effect of the background stray light on the detection result is large. Suppose there is a filter whose bandwidth is $5.5 \mathrm{~nm}$, that is, $\Delta \lambda=5.5 \mathrm{~nm}$. If the wavelength $\lambda=532 \mathrm{~nm}$ is calculated, the corresponding band width is:

$$
\Delta f=\frac{\Delta \lambda \cdot C}{\lambda^{2}}=5.8 \times 10^{10} \mathrm{~Hz}
$$

Since then, using a filter which has a very good performance, the calculated band is still very wide. But in the heterodyne detection system, the difference frequency bandwidth of the vibration light and the signal light can be selected as the frequency bandwidth of the information processor:

$$
f_{I F}=\left|f_{S}-f_{L}\right|
$$

$f_{S}$ is the frequency of the signal light, $f_{L}$ is the frequency of the vibration light, $f_{I F}$ is the frequency difference of the two beams of light. When the vibration light mixes with the signal light, stray light outside the difference frequency bandwidth will be filtered

4) Higher signal to noise ratio(SNR) can be obtained.

In the optical heterodyne detection, in order to obtain a higher signal to noise ratio, the corresponding local oscillator power is needed. The signal to noise ratio is:

$$
S N R=\frac{P_{I F}}{P_{N}}=\frac{2 \alpha^{2} P_{S} P_{L} M^{2} R_{L}}{2 M^{2} q e\left[\alpha\left(P_{L}+P_{S}+P_{B}\right)+i_{D}\right] \Delta B R_{L}+4 K_{B} T \Delta B R_{L}}
$$

In the formula, $P_{N}$ is the total noise power, $P_{B}$ is the background noise power, $T$ is the absolute temperature, $M$ is the photoelectric detector gain, $\Delta B$ is the detector bandwidth, $i_{D}$ is the dark current of the photoelectric detector, $K_{B}$ is Boltzmann's constant.

For the signal to noise ratio formula given by the formula (15), in order to get a high SNR, the noise caused by the vibration light needs to be much greater than the other noise components, and the noise part only considers the thermal noise and shot noise. Then the best SNR is:

$S N R=\frac{\eta_{D} P_{S}}{h v \Delta B}$

Wherein, $\eta_{D}$ is the quantum efficiency of the photodetector? As can be seen from the formula (16), to improve the signal to noise ratio, the power of the vibration light can be increased to suppress other noise components. But it does not mean that the power of this vibration can be infinite. When the power of this vibration is too high, it will lead to a large amount of shot noise, which can also cause a decrease of the SNR. Therefore, in order to improve the signal to noise ratio, the appropriate local oscillator power should be selected. 


\section{Conclusions}

In this paper, the principle, advantages and realization conditions of laser heterodyne detection are described in detail. On the basis of the specific implementation scheme of the laser heterodyne detection using laser radar, the traditional heterodyne detection is improved. Using all fiber system, the laser heterodyne detection system using all-fiber laser radar is designed by connecting the laser, the optical system, the receiving device and so on via the passive component. Finally, the performance of the all-fiber laser heterodyne detection system is analyzed. The results show that the system has a strong ability to detect weak signal, high conversion gain and SNR, and a good filtering effect. The system is very suitable for the application in laser ranging and so on.

\section{References}

[1] B. Koch, "Status and future of laser scanning, synthetic aperture radar and hyperspectral remote sensing data for forest biomass assessment", [J]. Isprs Journal of Photogrammetry \& Remote Sensing, no. 6, (2010), pp. 581-590.

[2] C. Qiwei, S. Gang and P. Hewei, "Large-size topography scanning with laser radar", [J]. Spacecraft Environment Engineering, (2012).

[3] B. Koch, "Status and future of laser scanning, synthetic aperture radar and hyperspectral remote sensing data for forest biomass assessment", [J]. ISPRS Journal of Photo grammetry and Remote Sensing, vol. 65, no. 6, (2010), pp.581-590.

[4] F. Qiang, J. Hui-lin and W. Xiao-man,"Research status and development trend of space laser communication", [J]. Chinese Optics, (2012).

[5] J. Dai ,B. Li and S. Zhang, "Intensity-optimized dithering technique for three-dimensional shape measurement with projector defocusing", [J]. Optics and Lasers in Engineering, no.53, (2014), pp.79-85.

[6] N. L. Karpinsky, M. D. Hoke and V. Chen, "High-resolution, real-time three-dimensional shape measurement on graphics processing unit", [J]. Optical Engineering, (2014).

[7] D. Chiang, "A new mobile and portable scanning lidar for profiling lower troposphere", [J]. Geoscientific Instrumentation, vol. 4, no. 1, (2014), pp. 165-190.

[8] N. Ming, "TheResearchof Wheel-flangeMeasurement System BasedonOptical Triangulation, Harbin University of Science and Technology”, [J], vol.15, no. 2 , (2010) Apr., pp. 39-42.

[9] J. Yan-shu, "Researchon Application of Analog-probefor Measuring Cente, Harbin University of Science and Technology", [J], vol.14, no.4, (2009) Aug., pp. 121-124.

[10] Z. Jianjun, "Research on Measurement and Parametric Assessment of the 3D Surface Microcosmic Topography, Harbin University of Science and Technology”, [D]. (2008) March, pp. 23-28. 
International Journal of Signal Processing, Image Processing and Pattern Recognition Vol. 9, No. 5 (2016) 